\title{
Large-Scale Mobile Multimedia Service Management in Next Generation Networks*
}

\author{
Daniel Negru ${ }^{1}$, Ahmed Mehaoua $^{1}$, Anastasios Kourtis $^{2}$, and Eric LeBars ${ }^{3}$ \\ ${ }^{1}$ CNRS-PRiSM Lab., University of Versailles, \\ 45, avenue des Etats-Unis 78035 Versailles, France \\ $\{$ dan, mea\}@prism.uvsq.fr \\ ${ }^{2}$ Institute of Informatics and Telecommunications NCSR "DEMOKRITOS", \\ Agia Paraskevi Attikis, 15310 Athens, Greece \\ kourtis@it.demokritos.gr \\ ${ }^{3}$ Thales Broadcast \& Multimedia \\ 1, rue de l'Hautil, zone des Boutries, 78700 Conflans Ste Honorine, France \\ Eric.lebars@thales-bm.com
}

\begin{abstract}
Last decade has seen a tremendous growing interest in wireless and mobile multimedia IP networking. As a consequence, the number and variety of devices allowing an Internet access has grown impressively and easilytransportable mobile devices have received an enormous success. More and more, new products are launched with an Internet access possibility and the trend now is the ability to get connected anywhere, anytime, anyhow. But, achieving such a task implicates an increasing complexity of the networking and service management. The fundamental multimedia applications, for which multicasting is the predominant delivery technique, need to be provided in a mobile environment. In this paper, we investigate the issues arising from the interoperation of mobile and multicasting mechanisms, focusing on specific multimedia constraints. From then, we propose an innovative protocol for an efficient delivery of multimedia services to desired mobile terminals. A solution for an anywhere, anytime, anyhow connection is hence presented.
\end{abstract}

\section{Introduction}

For many years now, there has been a sensational increase in the number and variety of devices connected to the Internet. Consequently, this has caused a lack of available IP addresses, one of the main issues addressed in Internet Protocol version 6 (IPv6) [1], the new version of the Internet Protocol, which is expected to be the leading standard for next generation networks. Above all, with the emergence of mobile devices such as laptops and PDAs, the number of mobile users, who expect to access services and applications the same way they did in wired networks, became larger and larger. The IETF proposed the Mobile IPv6 [2] approach to deal with this emerging network service requirement.

In the meantime, there has been a tremendous growing interest in multimedia applications. Demand for multimedia group communication, audio and video streaming, videoconferencing, distributed games or Internet TV has rapidly increased. The inher-

This work has been partially performed within the context of the European research project IST ATHENA (http://www.ist-athena.org). The authors would like to thank the participants for their contributions. 
ent nature of most multimedia applications is that a communication may include a large number of participants. They are considered as one-to-many or many-to-many, where one or multiple sources are sending to multiple receivers. Multicasting is the technique that most efficiently supports this type of transmission.

The combination of mobility and multicasting for the delivery of large-scale multimedia applications, in IPv6 networks, represents an important challenge. The principal objective is to enable users to access the desired multimedia program with sufficient Quality of Service anywhere, anytime and anyhow, hence through any kind of mobile device. There exists some techniques designed to multicast mobility, the most famous being IETF's ones based on Mobile IPv6 [2]: Bi-directional Tunneling (BT), which builds a tunnel between the home network and the foreign network of the mobile node, and Remote Subscription $(R S)$, which reconfigures the multicast tree by considering the new location of the mobile node. Several others have been elaborated but none has really addressed the problem focusing on IPv6 networks and specific multimedia constraints.

In this paper, we propose a novel approach for the management of IPv6 mobile terminals whishing to receive multicast multimedia services. We essentially focus on multimedia specific constraints and try to provide the best mechanism with the least delivery delays and service interruption periods. The result is a called Mobility for Multicast Multimedia Applications in IPv6 Networks (M3IP6), based on MIP6 with enhanced messages and addition of special entities. This protocol has been fully evaluated and will be deployed at a large-scale, thanks to the European research project ATHENA, for which it has received strong support.

The article is constructed as follows. First, we present related works, including Mobile IPv6's based solutions. Next, we focus on our proposal, by first stating the problem and then explaining the design and description of the M3IP6 protocol. After that, the evaluations of our solution, along with protocols' comparisons, are exposed. Finally, the large-scale deployment thanks to the ATHENA European project is presented.

\section{Related Works}

Some researches exist on the convergence of multicasting and mobility but they mainly focus on IPv4 networks. Besides Mobile IPv6 and its two extended approaches: Remote Subscription (RS) and Bi-directional Tunneling $(B T)$, there aren't many applicable to IPv6 networks.

\subsection{Mobile IPv6}

The first approach to multicast mobility in IPv6 networks would be to extend IETF's Mobile IPv6 protocol. In Mobile IP [3], each mobile node has a permanent home address (HoA), and receives a transient care-of address $(\mathrm{CoA})$ when visiting a foreign network. The mobile node registers its current CoA with its home agent (HA), which is located in its home network. The home agent intercepts and tunnels to the CoA all packets destined to the mobile node. Mobile IPv6 integrates new features: no more foreign agents needed, stateful or stateless autoconfiguration, route optimization, HoA Destination Option. Further information can be found in [2]. It appears that MIP6 is lighter and more efficient than MIP4. 
The current IETF's Mobile IPv6 specification proposes two approaches for supporting multicast services to mobile hosts: Remote Subscription (RS) and Bidirectional Tunneling (BT).

In Remote Subscription, the Mobile Node joins the multicast group each time it enters a foreign network. The main advantage of this approach is that multicast data are delivered on the shortest path. On the other hand, the multicast delivery tree must be frequently updated. This generates significant signaling overhead, and might result in unnecessary bandwidth consumption. Nevertheless, multicast traffic keeps flowing, until soft-state multicast group management information expires. Another major drawback of this proposition would be the join latency, which could be much too significant for multimedia real-time applications.

In Bi-directional Tunneling, the Home Agent forwards multicast packets to the Mobile Node through a unicast tunnel. This approach has the advantage that the multicast delivery tree is not updated every time the Mobile Node moves to a different network. On the other hand, the data delivery path is not optimal because of triangular routing and redundancy may occur in case of several Mobile Nodes willing to receive the same multicast flow, in the same foreign subnet: Tunnel Convergence Problem.

\subsection{Mobile Multicast Protocol (MoM) and Range-Based (RB)MoM}

Mobile Multicast Protocol (MoM) [4] solves the Tunnel Convergence Problem by using a Designated Multicast Service Provider (DMSP). This solution allows to provide at most once multicast delivery. It does solve the problem for IPv4 networks but not for IPv6 ones, since there are no foreign agents in IPv6. Also, a long DMSP handoff can occur if the FA has to reselect its DMSP, and multicast delivery may be disrupted, which is unacceptable for real-time communications.

The Range-Based MoM (RBMoM) [5] solution is a trade off between the shortest delivery path and the overhead induced by the multicast delivery tree reconfiguration. It uses Multicast Home Agents (MHA) with a limited service range to alleviate the problem of a long handoff. As for MoM, RBMoM uses FA and thus cannot be directly extended to IPv6. Also, the performance of $R B M o M$ is controlled by the selection of service range and, still, handoffs may occur when moving fast from high-distant networks. Other similar approaches can also be found, dedicated exclusively on IPv4, such as Mobility by Multicast Agent (MMA) [6].

\section{Proposal of an Efficient Protocol}

\section{for Mobile Multicast Multimedia Applications in IPv6 Networks}

Our solution for an efficient management of terminal mobility for multicast multimedia applications in IPv6 networks is now depicted. First, the problem of an efficient handling of multimedia communications in a mobile environment is stated through exposing the constraints of those specific services. Next, the architectural scheme of our solution is explained.

\subsection{Multimedia Services Constraints}

As presented above, no efficient solution exists for handling mobility for multicast applications in IPv6 networks, especially concerning multimedia sessions, which have 
their own constraints. Several proposals may achieve some parts of these. Nevertheless, they are not suitable to multimedia applications since they do not overcome multimedia constraints and especially in next generation's based IPv6 networks. Those constraints mainly include:

- Packet loss. Losses of multimedia packets can severely hamper the quality of the stream and damage the perception of the media at the end user;

- Delay and error resilience. Especially streaming video must reconcile the conflicting constraints of delay and error resilience. In order to maintain a high level of user interactivity, delay must remain relatively small (200ms);

- Delay variation or network jitter. An important delay variation would cause problems for applications that want to play out received data at a constant rate, such as streaming applications;

- Bandwidth variation. Available bandwidth varies with time and the streaming system should adjust its sending rate as well as the quality of the transmitted bitstream in accordance with these changes. This constraint cannot be overcome through our work. It is dedicated to the multimedia source.

The proposal described herein takes highly into consideration the first three constraints. Their evaluation will be processed trough two special metrics: service interruption period and delivery delay.

\subsection{Mobility for Multicast Multimedia Applications in IPv6 Networks (M3IP6)}

The proposed solution is called Mobility for Multicast Multimedia Applications in IPv6 Networks (M3IP6). It is based on IETF's Mobile IPv6 approach and is not dependent of any multicast protocol, as long as $M L D$ messages are used for registration, de-registration, and other important features at a LAN level. The different phases of the protocol will now be described.

First, a new Home Agent (HA) entity has to be considered for multicast sessions, in addition to the original HA, which task is dedicated to unicast flows. This Multicast Home Agent, MHA, will be in charge of retransmitting multicast flows to the Mobile Node when it is away from its home subnet. This is considered as phase 1 of the protocol. The MHA is dynamic; it depends on the previous location of the MN and changes accordingly.

The MHA and the HA are the same when the MN moves away from its home subnet, for the first time. But next, if the MN starts moving again to another foreign network, the new MHA will then not be the same entity as the HA. For most convenience, it should be the Designed Router (DR) of the last visited network but, it could also be another node of this network as well. The only imperative point is that the MHA has registered to all the multicast groups the MN has, so that it can retransmit the flows to the MN without having to register again - that's why the DR is certainly the most appropriate one. Therefore, when moving to foreign subnets, there's no significant latency at the MN side concerning multicast flows, essential feature for multimedia applications.

Also, while at a foreign network, a MN will subscribe to desired multicast groups by sending appropriate $M L D$ report messages onto the foreign network link, as described by remote subscription solution. After this phase is completed and multicast flows arrive from the DR of the link the $\mathrm{MN}$ is actually on, the $\mathrm{MN}$ will deregister from its MHA (which was on the previous visited link). Phase 2 of the M3IP6 proto- 
col is then initiated. We can see that the advantages of remote subscription are still present in our solution, without the drawback of long-lasting time wasting for the joining to (a) delivery tree(s).

We could have chosen not to specify a new entity, the MHA, and to make all the traffic (unicast and multicast) pass through the HA. Even though this approach would have seemed simpler, the HA being constantly considered as the multicast forwarding entity, it has too important drawbacks. Above all, this method is not efficient enough in case of multimedia streams. Concerning disruption, it is acceptable since the MN will only stop receiving multicast data for a short period of time, which corresponds to the delay introduced by the $\mathrm{MN}$ for sending the appropriate instructions to the HA and to trigger the resumption of multicast forwarding. Though, there still remains a huge problem in the case of many multicast registrations of Mobile Nodes. The HA will have to support all of these and thus congestion in the node can occur, especially with multimedia streams, which are usually very heavy. The HA would be overloaded. Therefore, this method is not adapted at all to large-sized mobile nodes environment and high-consuming bandwidth applications.

\subsection{M3IP6 Protocol Description for Terminal Mobility}

The M3IP6 protocol is based on extensions of Mobile IPv6 and MLD proposals.

New messages are added to the Mobile IPv6's ones:

- Multicast Binding Update (MBU) from the MN to its MHA, to notify that it has moved;

- Multicast Binding Acknowledgement (MBA), return message from the MHA after receiving a MBU;

- Deregistration MBU (lifetime=0) and MBA, messages between the MN and its MHA to cease the retransmitting of multicast flows through this way.

Some additional lists will be implemented into the nodes:

- Multicast Home Agents list for MNs, similar to the Home Agents list for unicast flows;

- Multicast Binding Cache for MHAs, similar to the Binding Cache for unicast flows.

For $M L D$ extensions, only a special message is added to inform the Designed Router that it should not stop receiving the multicast flow, provided the MN is the last one willing to receive it and it is away: MLD Listener Hold. Here is a detailed description of how this protocol works for terminal mobility:
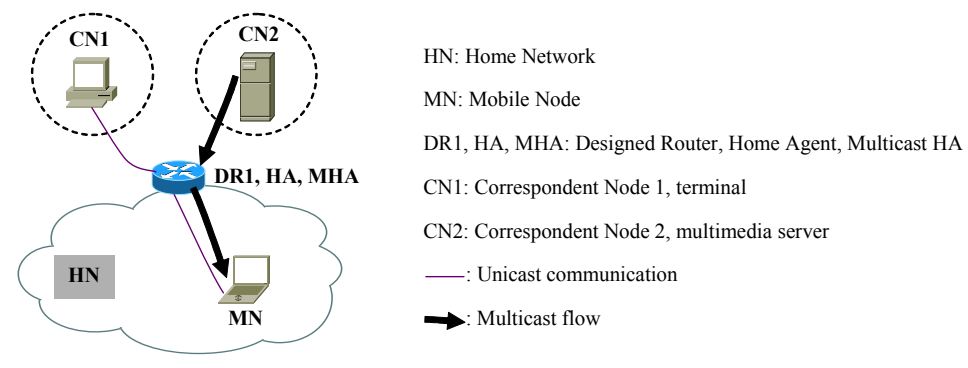

Fig. 1. MN at Home Network. 
First, the $\mathrm{MN}$ is at home (Fig. 1). It establishes an unicast communication with the terminal $\mathrm{CN} 1$ and it receives a multicast flow from the multimedia server CN2. The Designed Router is also determined as the HA of the MN.

Next, the MN moves to a foreign network (Fig. 2). Concerning the unicast communication between the MN and CN1, classic Mobile IPv6 messages are sent to establish the tunnel MN-HA: Binding Update and Binding Ack. The same mechanism is used for the multicast flow from the multimedia server $\mathrm{CN} 2$, to which the $\mathrm{MN}$ is registered: Multicast $\mathrm{BU}$, from the $\mathrm{MN}$ to the $\mathrm{MHA}$ (which is the same entity as the HA at this moment) and Multicast BA, from the MHA to the MN. Then, the Multicast Binding Cache of the MHA is updated consequently with the Care-of-address of the MN and a Multicast MHA-MN tunnel is established. In this first move case, we consider the tunnel as the same as for unicast communication. This represents phase 1.

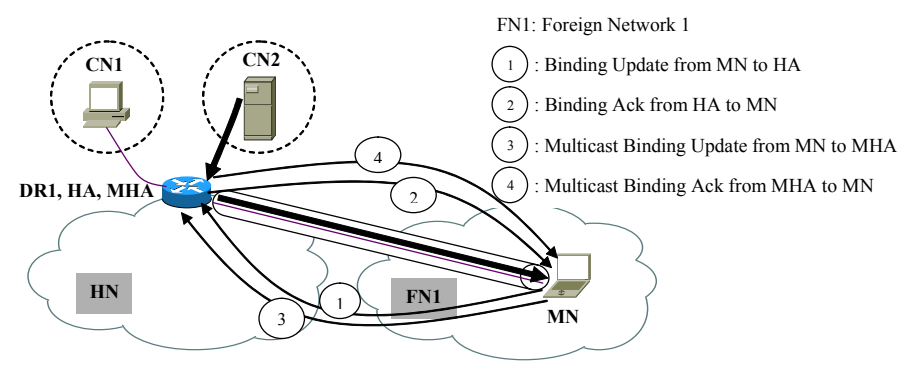

Fig. 2. First Move of the MN.

After the reverse tunnels are established between the HA or MHA, and the MN for the unicast and multicast applications, packets are being encapsulated and transmitted through this way. Next (Fig. 3), for the unicast communication, route optimization is established according to the Mobile IPv6 specifications. Concerning the multicast flow, the MN registers to the DR2 of the foreign network it is actually in. When the registration process is done and the $\mathrm{MN}$ may receive the multicast flow from DR2, it informs its MHA that it does no longer need to receive these packets through the tunnel: Multicast Deregistration BU from the MN to the MHA (1), Multicast Deregistration Back from the MHA to the MN (2). Then, the multicast MN-MHA tunnel is destroyed; the MN-HA tunnel for unicast communications remains still (as described in Mobile IPv6's draft). The MHA is no longer DR1 but it switches to DR2. From now on, phase 2 of the mechanism is activated.

Now, the MN starts moving again to another foreign network FN2 (Fig. 4). The same process as above is initiated (phase 1) but at this point, the MHA and the HA are not the same anymore. Concerning the multicast flow between $\mathrm{CN} 2$ and the MN, first a MBU is sent from the MN to the MHA, updating consequently its Multicast Binding Cache with the new CoA of the MN. The tunnel between the MHA and the new location of the $\mathrm{MN}$ is then established. Multicast packets from $\mathrm{CN} 2$ are encapsulated and transmitted this way.

Afterwards, the same process as the one explained in Fig. 3 is executed again. The MN enables Route Optimization for the unicast communication and remote subscription to the multicasting flow through the new DR for multicast communications. With this solution, MNs will always act this way when switching from a subnet to another, even when they come back home. 


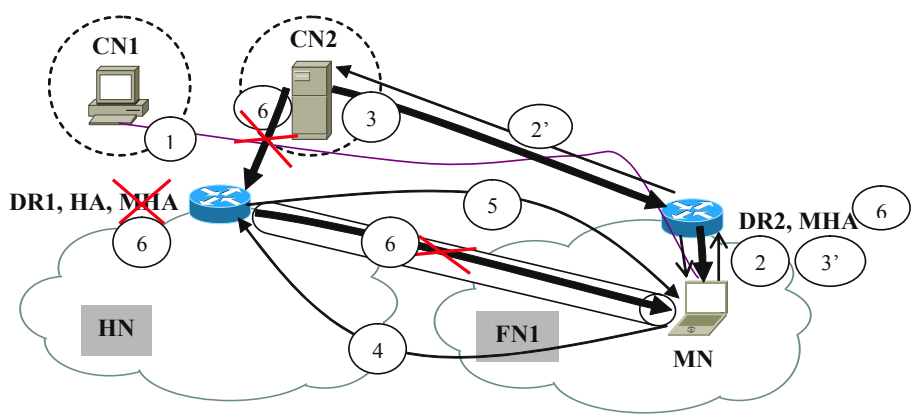

(1) : Route Optimization Establishment for Unicast Communication

(2) : Multicast Registration Procedure through DR2 (MLD and PIM messages)

(3): Multicast Flow reception through DR2

(4) : Multicast De-registration BU from $\mathrm{MN}$ to MHA

(5) : Multicast De-registration BAck from MHA to MN

6 : Suppression of the Multicast Flow from the MN-MHA tunnel, suppression of MN-MHA tunnel and establishment of DR2 as the new MHA for MN.

Fig. 3. Optimization of Mobile O7perations.

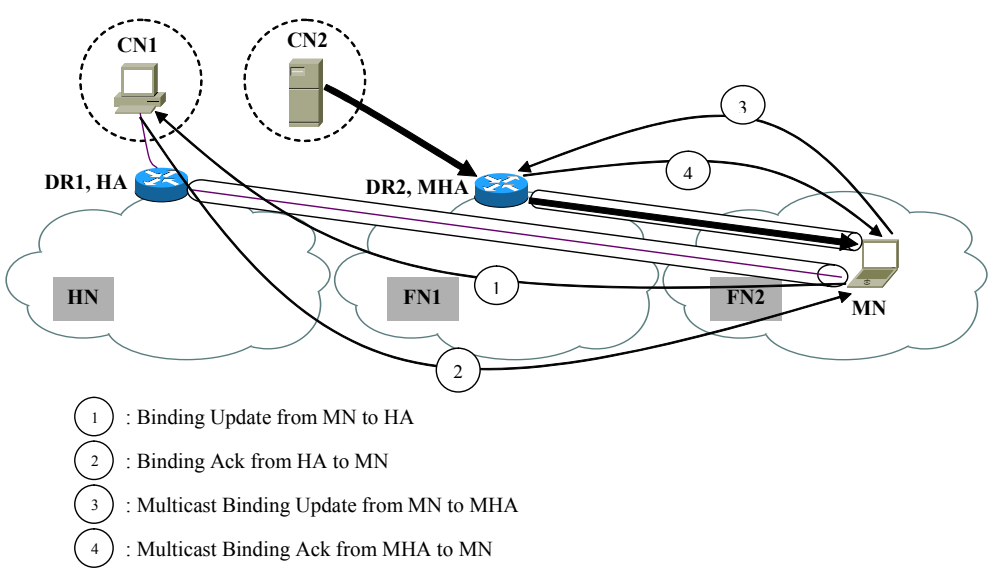

Fig. 4. MN's Next Moves Process.

\section{Evaluation and Demonstration}

Our solution is well-adapted for supporting multimedia applications since it significantly reduces disruption and insures optimal multicast routing and low system overload. The M3IP6 protocol has been fully evaluated when dealing with terminal mobility through specific metrics, comparison, and simulation. The implementation and demonstration stage has found support in the European project ATHENA. 


\subsection{Protocol's Evaluation}

The evaluation of our protocol has been performed first through a theoretical evaluation based on metrics, then based on a comparison to other mobile multicast protocols. There is also a simulation process and deeper looks that can be found in [10].

Theoretical Evaluation Based on Metrics. We compare M3IP6 with the two solutions already proposed in Mobile IPv6: Bi-directional Tunneling (BT) and Remote Subscription (RS). For efficient evaluation, we use two special metrics: the service interruption period and the delivery delay. Service interruption is fundamental for the evaluation of packet losses and disruptions, one of the important constraints of multimedia applications. Delivery delay also highly concerns real-time applications, for which important delays imply loss of quality. Hence, we suppose the followings: $d(a, b)$ : Distance between node $a$ and node $b$, in number of links; $S$ : The multicast source; $D l_{p r o p}$ : Propagation delay on a link, assuming it is the same on each link of the network for each kind of message and neglecting processing and queuing delays; $\operatorname{Int}(M N)$ : Service interruption or the time interval during which the mobile node looses multicast connectivity, when it moves from its home network to a foreign one; $\operatorname{Del}(M N, S)$ : Delivery delay or the time interval necessary to deliver a multicast packet from the source $\mathrm{S}$ to the mobile node. $J \operatorname{Del}(M L D)$ : The Join Delay, experienced by $M L D$ timers. This value could be set to a lower one but this may have repercussions (overflow of signalization). By default, it is of $125 \mathrm{~s}$.

Service Interruption Period. Concerning the Service Interruption Period, we compare the three solutions by neglecting agent discovery and we reach the following conclusion. All details can be found in [10]. We obtain the shortest service interruption period with our protocol:

$$
\begin{aligned}
& \operatorname{Int}_{\mathrm{M} 3 \mathrm{IP6}}(\mathrm{MN})<\operatorname{Int}_{\mathrm{BT}}(\mathrm{MN})<<\operatorname{Int}_{\mathrm{RS}}(\mathrm{MN}) \\
& \text { if } \mathrm{d}\left(\mathrm{MN}_{\mathrm{CoA}}, \mathrm{MHA}\right)<\mathrm{d}\left(\mathrm{MN}_{\mathrm{CoA}}, \mathrm{HA}\right) .
\end{aligned}
$$

Or at least, the same as for $B T$ in some cases (when the $M N$ moves for the $1^{\text {st }}$ time):

$$
\operatorname{Int}_{\mathrm{M} 3 \mathrm{IP}}(\mathrm{MN}) \sim \operatorname{Int}_{\mathrm{BT}}(\mathrm{MN})<<\operatorname{Int}_{\mathrm{RS}}(\mathrm{MN}) .
$$

Delivery Delay. We now consider and compare the Delivery Delays of the three solutions. We neglect processing and queuing delays of the messages.

In $B T$, there is the establishment of a tunnel between the HA and the MN. We will not consider the encapsulation and decapsulation time of the packets:

$$
\operatorname{Del}_{\mathrm{BT}}(\mathrm{MN}, \mathrm{S})=\operatorname{Del}_{\text {prop }}\left[\mathrm{d}(\mathrm{S}, \mathrm{HA})+\mathrm{d}\left(\mathrm{HA}, \mathrm{MN}_{\mathrm{CoA}}\right)\right] \text {. }
$$

The packets do not have a direct path, as shown Fig. 5 (a), consequently a delay occurs in the delivery mechanism. In $R S$, there is no tunnel and therefore, the delivery is achieved directly, no delay is induced; it can be seen in Fig. 5 (b):

$$
\operatorname{Del}_{\mathrm{RS}}(\mathrm{MN}, \mathrm{S})=\operatorname{Del}_{\text {prop }} \mathrm{d}\left(\mathrm{S}, \mathrm{MN}_{\mathrm{CoA}}\right) \text {. }
$$

However, in M3IP6, it is a bit more complex to estimate the Delivery Delay. The first phase of the M3IP6 mechanism consists of the establishment of a bi-directional tunnel between the MHA and the MN. The Delivery Delay could be compared to the one of $B T$, with the difference that it is not the HA but the MHA that retransmits the multicast flow: 


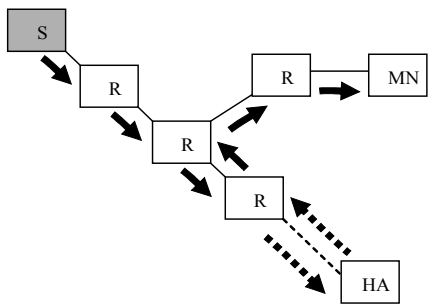

(a) Data Delivery in BT

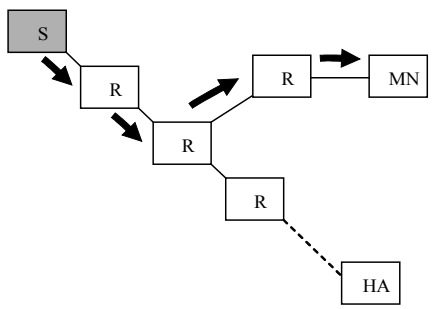

(b) Data Delivery in RS

Fig. 5. Data Delivery Mechanisms in BT and RS.

$1^{\text {st }}$ phase: $\quad \operatorname{Del}_{\mathrm{M} 3 \mathrm{IP6}}(\mathrm{MN}, \mathrm{S})=\operatorname{Del}_{\text {prop }}\left[\mathrm{d}(\mathrm{S}, \mathrm{MHA})+\mathrm{d}\left(\mathrm{MHA}, \mathrm{MN}_{\mathrm{CoA}}\right)\right]$.

Thus, since the MN's movement will not be, in most cases, further than one network away at a time, only one (or at most two) router(s) will generally separate the two networks, as shown Fig. 6.

Consequently, on the first stage of the mechanism, the delivery delay is more optimal for M3IP6 than for BT (and of course, a bit less optimal than $R S$ ), in most cases:

$$
1^{\text {st }} \text { phase: } \quad \operatorname{Del}_{\mathrm{RS}}(\mathrm{MN}, \mathrm{S})<\operatorname{Del}_{\mathrm{M} 3 \mathrm{IP6}}(\mathrm{MN}, \mathrm{S})<\operatorname{Del}_{\mathrm{BT}}(\mathrm{MN}, \mathrm{S}) \text {. }
$$

When reaching the second phase, the $\mathrm{MN}$ is registered to multicast flows through the DR of the visited network, exactly identical to the $R S$ mode. Therefore, the delivery of the packets is direct:

$$
2^{\text {nd }} \text { phase: } \quad \operatorname{Del}_{\mathrm{M} 3 \mathrm{IP6}}(\mathrm{MN}, \mathrm{S})=\operatorname{Del}_{\text {prop }} \mathrm{d}\left(\mathrm{S}, \mathrm{MN}_{\mathrm{CoA}}\right) \text {. }
$$

We then obtain, for the second phase, the best delivery delay possible with our protocol:

$2^{\text {nd }}$ phase: $\quad \operatorname{Del}_{\mathrm{M} 3 \mathrm{IP6}}(\mathrm{MN}, \mathrm{S})=\operatorname{Del}_{\mathrm{RS}}(\mathrm{MN}, \mathrm{S})<\operatorname{Del}_{\mathrm{BT}}(\mathrm{MN}, \mathrm{S})$.

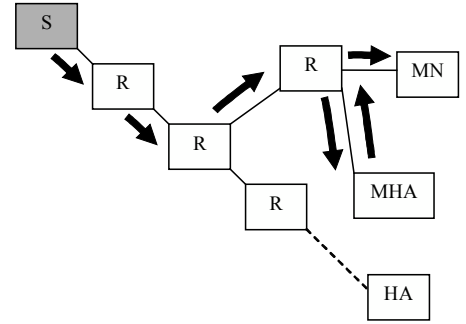

(a) Data Delivery in M3IP6 $\left(1^{\text {st }}\right.$ phase $)$

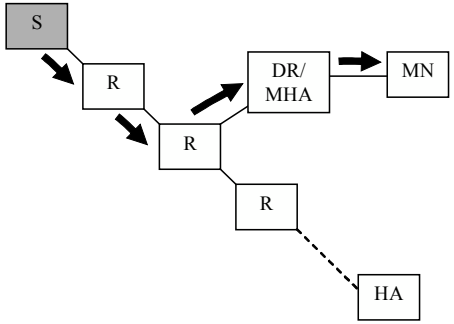

(b) Data Delivery in M3IP6 $\left(2^{\text {nd }}\right.$ phase $)$

Fig. 6. Multicast Data Delivery Mechanism in M3IP6.

In conclusion, our M3IP6 protocol is the most efficient one, considering the two most important metrics for multimedia applications: the Service Interruption Period and the Delivery Delay. The shortest interruption period is obtained, as well as the best delivery delay, compared to $R S$ and $B T$.

Comparison to Other Mobile Multicast Protocols. The main advantage of our solution is that it is entirely designed to support for the best multimedia applications, focusing on their constraints. Even if it may increase the bi-directional tunnel duration, 
it absolutely minimizes the multicast tree complexity while still meeting the delay constraints (i.e. a short handoff for services continuity). The use of a Multicast Home Agent (MHA) reduces significant disruption and the establishment of the multicast subscription at the visited network side assures optimal multicast routing and low system overload. Moreover, this distinct location of MHA and HA allows processing of multicast flows by a QoS differentiation scheme, for which solutions already exist. Minimum latency, short join delay, routing optimality are among the important features the proposal has been based on. System load has also been taken into account, as well, in this evaluation process.

The following table (Table 1) shows the advantages and drawbacks of most of the proposals in this area: Remote Subscription $(R S)$, Bi-directional Tunneling $(B T)$, Range-Based MoM (RBMoM), MMA, and ours, among different criteria.

Table 1. Comparison of Mobile Multicast Protocols.

\begin{tabular}{|l|l|l|l|l|l|}
\hline & RS & BT & RBMoM & MMA & M3IP6 \\
\hline Join Delay & High & Optimal & Short & Short & Optimal \\
\hline Multicast Routing & Optimal & $\begin{array}{l}\text { Non- } \\
\text { Optimal }\end{array}$ & Sub-Optimal & Sub-Optimal & $\begin{array}{l}\text { Sub-Opt.- } \\
\text { >Opt. }\end{array}$ \\
\hline Handoff Occurrence & High & Optimal & Sub-Optimal & Low & Optimal \\
\hline Tunnel Convergence & No & Yes & No & No & No \\
\hline IPv6 Support & Yes & Yes & No & No & Yes \\
\hline Add. Signalisation & No & No & Yes & Yes & Yes \\
\hline Add. Support at FN & Low & No & No & Low & Yes \\
\hline System Load & No & Yes & Low & Low & Low \\
\hline Source Mobility & Possible & Possible & Not Specified & Not Specified & Possible \\
\hline
\end{tabular}

\subsection{Demonstration on ATHENA Platform}

The EU-funded IST Project ATHENA (ATHENA - Digital Switchover: Developing Infrastructures for Broadband Wireless Metropolitan Area Network Access [9]), which started in January 2004, takes into consideration mobility concepts and technology interoperation between DVB-T and IP. ATHENA proposes the use of the DVB-T in regenerative configurations and exploits the networking capabilities of the television stream for the creation of a powerful backbone that interconnects distribution nodes within a city. Fig. 7. shows an overall representation of the networking aspects of the project.

Among its objectives, the ATHENA European project is conducting research activities in DVB-T system and mobility. One of the goals of this research project is to set the proposed architecture described above and make feasible scenarios of mobility, with proper solutions and enhancements. In this particular environment, we can distinguish several mobility issues, either on the concern of access networks behind CMNs (WLAN and UMTS devices), or switching between CMNs and experiencing layer-3 handovers and finally, at a larger scale between broadcasting areas. Concerning access networks behind CMNs, such as WLAN and UMTS, solutions for achieving mobility in those cases are known and exploited. The first challenge is to support the mobility case when a mobile user equipped with a DVB-T receptive device 
switches from one broadcasting area to another or inside the same broadcasting area from a frequency to another. A second objective is to permit the accessibility of IP services by no DVB-T receivers, located behind CMNs and able to perform mobility actions from one $\mathrm{CMN}$ to another. This point is covered by our proposed protocol: M3IP6. In this broadcasting context, there will essentially be multicast multimedia IP services, which will be transmitted between users. The intention is to provide wide access to mobile users, permitting them to switch instantly from an area to another.

Therefore, the ATHENA project consists of a perfect support for integrating and developing at a large scale, mobility aspects, issues and, thus, our proposed solution.

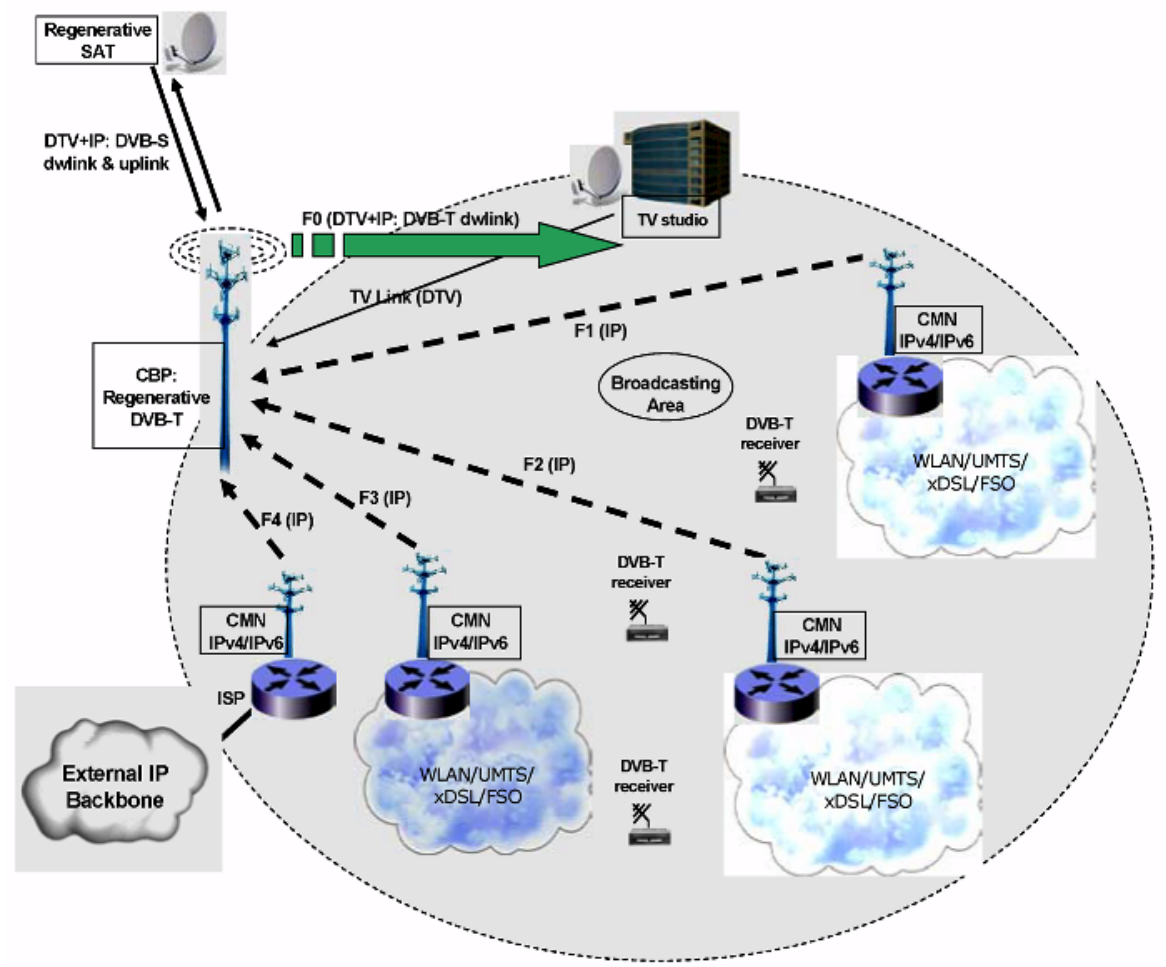

Fig. 7. Overall ATHENA Network Architecture.

\section{Conclusion}

The establishment of an efficient handling of mobility for multicast multimedia applications becomes a need nowadays, especially given the tremendous growing demand for these kinds of services and the steady increase in the number of mobile wireless devices connected to the Internet. Over the last few years, there have been several interesting proposals to achieve the interoperation of Mobility and Multicasting, but none explicitly focused on IPv6 networks. The IETF's Mobile IP Working Group proposes Remote Subscription and Bi-directional Tunneling but they both have important drawbacks, inadequate to the specific QoS required by multimedia applications. 
We introduced a novel approach for an efficient mobility management for Multicast Multimedia Services in IPv6 Networks and proposed the M3IP6 (Mobility for Multicast Multimedia Applications in IPv6 Networks) protocol. Our solution provides a simple and efficient method, based on Mobile IPv6, for transparently handling multicast receivers' mobility. M3IP6 advantages are to reduce data delivery delays and achieve almost a no service interruption period. Therefore, it overcomes most of the important multimedia constraints. Above all, it appeared to be designed to fully comply with multimedia communications. Its integration into the European project ATHENA will provide a perfect context for the deployment at a large scale of M3IP6's proposal.

\section{References}

1. Deering, S., Hinden, R.: Internet Protocol, Version 6 (IPv6) - Specification. RFC 2460 (1995)

2. Johnson, D., Perkins, C., Arkko, J.: Mobility Support in IPv6. Internet Draft, draft-ietfmobileip-ipv6-24.txt, work in progress (2003)

3. Perkins, C.: IP Mobility Support. RFC 2002 (1996)

4. Harrison, T., Williamson, C., Mackrell, W., Bunt, R.: Mobile Multicast (MoM) Protocol: multicast support for mobile hosts. Proceedings of ACM MOBICOM '97 (1997) 151-160

5. Lin, C.R., Wang, K.-M.: Mobile Multicast Support in IP Networks. Proceedings of IEEE INFOCOM'00 (2000) 1664-1672

6. Wang, Y., Chen, W.: Supporting IP Multicast for Mobile Hosts. ACM/Kluwer Mobile Networks and Applications, Special Issue on Wireless Internet and Intranet Access, vol. 6, no. 1 (2001) 57-66

7. Jelger, C., Noel, T.: Multicast for Mobile Hosts in IP Networks: Progress and Challenges. IEEE Wireless Communications Magazine, vol. 9, no. 5 (2002) 58-64

8. Feamster, N.G.: Adaptive Delivery of Real-Time Streaming Video. Ph.D. Thesis, Massachusetts Institute of Technology (2001)

9. IST ATHENA Report: ATHENA Digital Switchover: Developing Infrastructures for Broadband Access (2003)

10. Negru, D., Mehaoua, A.: Deploying Multimedia Services to Mobile Users in Next Generation IPv6 Networks. Technical Report PRiSM Lab., University of Versailles, France (2004) 